\title{
Linearized Kalman Filter aided Wavelet Transform with Adaptive Thresholding Methodfor ECG signal
}

\author{
Mythili Thirugnanam, P.Megana Santhoshi
}

\begin{abstract}
Cardiovascular diseases (CVD) are the most chronic and dangerous diseases in worldwide. The early prediction of CVD can help to prevent deaths due to these diseases, using bio-medical signal analysis. In this field, the ECG signal plays an important role due to its significant nature of providing the health-related information. However, the signal acquisition process is a crucial step where signals get corrupted due to electrode movement, muscle movement and other types of interference which can degrade the performance of the signal analysis. Several approaches have been introduced but achieving the desired performance robustly is still considered as a challenging task. This paper presents a novel approach for ECG signal filtering by combining a combination of the extended Kalman filter, wavelet transform and an adaptive thresholding approach called as Linearized Kalman Filter aided Wavelet transform with Adaptive Thresholding (LKFWAT). In this process, the initial states of the signal are observed using a Kalman filter, later; a linearization scheme is presented to represent the signal in the linear form. Finally, an adaptive threshold method is applied to reduce the noise during signal construction. It will show the significant improvement in next level process of disease classification. A comparative experimental analysis is carried out which shows that the proposed approach achieves improved performance when compared with the state-of-art ECG denoising techniques.
\end{abstract}

Keywords: ECG signal filtering, Kalman filter, Wavelet transform, Adaptive Thresholding, Signal artifacts.

\section{INTRODUCTION}

Medical health related complications are the most crucial issues in human life which affects the quality of living of human being. There are several chronic fatal diseases which can cause death to the patients, such as Cancer, Diabetes, Alzheimer's and cardiovascular diseases etc... A study presented in [1] shows that $54 \%$ portion of total deaths are caused due to these diseases in 2016. Generally, these diseases are caused due to unhealthy lifestyle, genetic inheritance and other risk factors. Cardiovascular diseases are considered most crucial and leading disease of cause of deaths. The world Health Organization (WHO) presented a

Revised Manuscript Received on October 22, 2019

* Correspondence Author

Mythili Thirugnanam*, Associate Professor Senior, School of Computer Science and Engineering (SCOPE), Vellore Institute of Technology, Vellore, Tamilnadu India Email: tmythili@vit.ac.in

P. Megana Santhoshi, PhD scholar, School of Computer Science and Engineering (SCOPE), Vellore Institute of Technology, Vellore, Tamilnadu, India Email: pmsanthoshi@gmail.com report which shows that, the Heart disease and stroke causes around 17 million deaths every year. Cardiac diseases are the most fatal and by 2020 it will the leading reason of deaths caused due to the diseases. It is estimated that CVD will cause around 20 million deaths and 24 million deaths by 2030[2].

Cardiac diseases include several diseases such as heart failure, stroke, hypertensive heart disease, rheumatic heart disease, cardiomyopathy (disease group which affects the heart muscle), and heart arrhythmia (irregular heartbeats) etc... In cardiac diseases, cardiomyopathy and arrhythmia are the most common diseases which can cause the death to the patient. Cardiomyopathy is a group of diseases which affects the heart muscle by changing the size and thickness of heart muscle whereas heart arrhythmia is a group of conditions which affects the heartbeat rate i.e. it causes irregularity in heart rate by making it too slow or too fast. In order to prevent the deaths due to these types of cardiovascular disease, preliminary diagnosis and early prediction strategies need to be developed which can help to the patient by taking the appropriate diagnosis and reducing the threat.

In this field, biomedical signals are considered as a promising solution to achieve the various types of physiological activities of organisms, including, gene sequencing, protein sequencing, neural rhythms, cardiac rhythms, tissues and organ signals. Biomedical signal processing is a technique to extract the useful information from the biomedical signals which can be used for biologists and physicals to monitor and diagnosis of the distinct diseases [3]. According to the bio-medical field, Electrocardiogram (ECG) signal processing is a widely adopted method for analyzing the heart conditions. A brief discussion about ECG signals is presented in subsection A. During last decade, several techniques of cardiac disease detection using ECG signal processing, have been developed. These techniques focus on the ECG signal filtering, ECG signal feature extraction and ECG signal classification. Generally, during the ECG signal acquisition, signals get contaminated due to various types of artifacts such as power line interference, noise, mechanical forces on electrodes and motion artifact which can degrade the quality of signal analysis. Hence, removal of these types of artifacts is a primary task in the ECG signal processing.

Based on this assumption, several approaches have been introduced such as EMD (Empirical Mode Decomposition) based baseline wander correction $[4,5,6]$, efficient recursive least-squares (RLS) adaptive notch filter with an optimal linear discriminant analysis (LDA) algorithm which helps to 
identify the presence of power line interference [7], Kalman Filtering [8,9], Wavelets [10, 11, 12], morphological filtering [13], adaptive Kalman filter [14], Chang and Liu [15] introduced a novel technique of Gaussian noise filtering from ECG signal using Wiener filter and ensemble empirical mode decomposition in this field of signal filtering, evolutionary computation optimization based schemes are also introduced for ECG signal denoising [16], Kabir and Shahnaz [17] presented a novel approach by combining the EMD and DWT (Discrete Wavelet transform) technique for ECG filtering, DWT \& Neural Network based adaptive scheme [18].

Similarly, several approaches of ECG signal feature extraction and classification are also presented. For feature extraction also, Wavelet based schemes plays important role [19]. Banerjee and Mitra [20] presented a cross wavelet based scheme for ECG classification, dual tree complex wavelet based feature extraction [21], adaptive threshold \& Principal Component Analysis (PCA) [22], Morphological and statistical features [23], non-linear feature extraction such as principal component analysis (PCA) and kernel independent component analysis (KICA) [24]. These features are used for generating the trained model for different types of classifiers. Several classification schemes are also present such as Deep Neural network [25], Neural Network [26], Support Vector Machine [27], and Naïve Bayes classifier [28] etc...

\section{A. ECG signals}

The electrocardiogram (ECG) signal record represents heart activity. These recordings are the quick and efficient way of diagnosing cardiovascular diseases. It enables remote monitoring of patients and facilitates telemedical applications. The recording procedure is safe, harmless and needs no supervision [9]. Though, there are plenty of portable devices available for ECG recording but still quality of signal corrupts due to unwanted interference such as muscle (EMG) noise, respiration (baseline wander) noise, poor channel, etc., [4]. To improvise the quality of ECG, several filtering methods have been proposed in the past that extract valid signal from the noisy signal.

This paper is organized as follows: section II presents a brief discussion about recent techniques in the field of ECG signal filtering and identifies the drawback in the existing techniques. Section III presents the proposed novel approach for ECG signal filtering, experimental study using proposed approach is presented in section IV and finally, section V presents the concluding remarks about the proposed approach and the performance.

\section{LiteratURE SURVEY}

This section presents a brief discussion about some recent techniques of ECG signal filtering which are developed for different type of cardiac diseases. In this field, the wavelet transform plays important role due to their significant nature of sub-band decomposition. Yadav [29] suggested that DWT based approaches are dependent on the inherit sparsity of clean signal in the transform domain hence these methods do not face issues of data sample estimation unlike the sample-based denoising algorithms [30]. However, the DWT method fails to exploit the non-redundancy information in the signal. Hence, Yadav [29] presented a combined approach using block based NLM (non-Local Means) and Wavelet Transform schemes. In an another study, Abbaspour, Gholamhosseini, and Linden [31] presented two methods for signal denoising, the first technique is developed wavelet transform based on non-linear thresholding and another method is developed by combining wavelet transform and ICA (Independent Component Analysis). Generally, these signals are contaminated due to baseline wander, power-line interference (PLI), muscle artifacts and motion artifacts. Singh and Sunkaria [32] discussed about the ECG signal filtering issues and developed a filtering approach using empirical wavelet transform (EWT) along with the adaptive filter theory for PLI cancellation.

Kumar, Singh, and Anurag [33] developed filter bank based model for ECG signal processing. The main aim of this approach is to reduce the reconstruction error during the filtering phase. In order to obtain this, a cosine modulated filter bank based approach is developed. Moreover, several adjustable filters such as Kaiser, Cosh and Exponential filters are also included to design the complete prototype of filter banks.

Similarly, Sharma, San Tan, and Acharya [34] developed an end-to-end automated process of ECG analysis and also presented filter bank approach for ECG signal analysis. In order to perform the desired task, bi-orthogonal filter banks are designed. These bi-orthogonal filter banks are later decomposed into two-band optimal bi-orthogonal filter bank which provides the analysis of low pass and half band filter. In this work, the filter design problem is considered as a convex optimization where signal reconstruction quadratic functions are considered as objective function. From the designed filter banks, the input ECG signals are decomposed into six sub bands which are later processed through the feature extraction and classification phase. Recently, Eminaga, Coskun, and Kale [35] focused on motion artifact removal from the ECG signals and developed FIR wavelet filter bank based method. The conventional FIR filtering methods suffer from the computational complexity issues hence, in this work hybrid approach of FIR/IIR is presented along with DWT filtering. This approach is design for reducing the time and complexity for ECG processor for hardware application. Based on wavelet tree, El B'charri, Latif, Elmansouri, Abenaou, and Jenkal, [36] presented a dual-tree wavelet transform scheme which relies on thresholding tuning for noise reduction.

Panigrahy and Sahu [37] developed a novel methodology for extracting the fetal electrocardiogram (FECG) from the single channel abdominal ECG with the help of differential

evolution approach, extended kalman smoother, and Neuro-fuzzy System model. 


\begin{tabular}{|c|c|c|c|}
\hline Reference no. & Technique & Noise Types & Observations \\
\hline [29] & DWT & AWGN & $\begin{array}{l}\text { Reconstruction redundancy is reduced } \\
\text { and preserves diagnostic features }\end{array}$ \\
\hline [30] & nonlocal means & Additive Noise & $\begin{array}{l}\text { Signal distortion is minimized but } \\
\text { works for low-SNR signals. }\end{array}$ \\
\hline [31] & Wavelets & Motion Artifacts & $\begin{array}{l}\text { Wavelet combination with ICA } \\
\text { provides better results }\end{array}$ \\
\hline [32] & $\begin{array}{l}\text { Empirical Wavelet } \\
\text { Transform }\end{array}$ & Power line Interference & $\begin{array}{l}\text { It requires total number of modes for } \\
\text { Empirical Mode Decomposition. }\end{array}$ \\
\hline [33] & Modulated Filter Banks & Quantization noise & $\begin{array}{l}\text { Distortion is reduced and this process } \\
\text { can be implemented for speech signals } \\
\text { also. }\end{array}$ \\
\hline [34] & $\begin{array}{l}\text { Bi-orthogonal Filter } \\
\text { Bank }\end{array}$ & Wander & $\begin{array}{c}\text { A complete classification system is } \\
\text { developed for ECG signal }\end{array}$ \\
\hline [35] & $\begin{array}{l}\text { IIR/FIR Wavelet Filter } \\
\text { Banks }\end{array}$ & $\begin{array}{c}\text { Baseline line wander and muscle } \\
\text { artifacts }\end{array}$ & $\begin{array}{l}\text { Less resource utilization and improved } \\
\text { power consumption }\end{array}$ \\
\hline$[36]$ & $\begin{array}{l}\text { Dual tree wavelet } \\
\text { transform (DT-WT) }\end{array}$ & $\begin{array}{l}\text { power-line interference, baseline } \\
\text { wander, } \\
\text { Muscle noise and motion artifact. }\end{array}$ & $\begin{array}{l}\text { This approach gives better results for } \\
\text { all types of noises. }\end{array}$ \\
\hline [37] & Kalman Filter & $\begin{array}{l}\text { baseline wander and the power } \\
\text { line interference }\end{array}$ & $\begin{array}{l}\text { Extraction of Fetal ECG from } \\
\text { abdominal ECG. }\end{array}$ \\
\hline [38] & Wavelet \& CEEMDAN & Random and baseline wander & $\begin{array}{l}\text { This technique can work with the } \\
\text { non-stationary and non-linear signals. }\end{array}$ \\
\hline [39] & Guided filtering & AWGN and Muscle artifact & $\begin{array}{l}\text { This can be used for complex noise } \\
\text { scenarios }\end{array}$ \\
\hline [41] & Fourier Transform & Baseline and power line & $\begin{array}{l}\text { Lower and simple computational } \\
\text { complexity }\end{array}$ \\
\hline [42] & Bayesian Approach & $\begin{array}{l}\text { power-line interference, baseline } \\
\text { wander, } \\
\text { Muscle noise and motion artifact. }\end{array}$ & Reduced computational cost \\
\hline
\end{tabular}

In this work, a pre-processing model is developed to reduce the noise from the ECG signal. During pre-processing stage, moving window median filtering scheme is applied to reduce the baseline wandering artifact in the ECG. $\mathrm{Xu}, \mathrm{Luo}, \mathrm{Li}$, and Song [38] presented a hybrid approach for ECG signal denoising by using complete ensemble empirical mode decomposition with adaptive noise (CEEMDAN) and wavelet threshold scheme. According to this process, the input signal is decomposed into multiple IMFs series which are later sorted from high frequency to low frequency. After sorting the data, the correlation is applied to identify the noisy segment in the decomposed bands. Finally, IMFs are denoised using wavelet thresholding scheme. Recently, Hao et al. [39] presented ECG signal denoising process using guided filtering scheme using a multi-lead scheme where a guided filter is adapted for denoising the signal. El Bouny, Khalil, and Adib [40] also presented CEEMDAN based approach for ECG signal denoising. According to this scheme, initially IMFs of input signal are computed and high order statistics is applied resulting in grouping of noisy and useful signals.

Bahaz and Benzid [41] focused on both power line interference and baseline wander (BW) artifacts in ECG signal analysis. According to this approach, first of BW signal is estimated and total number of low frequency bands which are contributing in BW formation, are achieve. In next level, base line drift is estimated and baseline shift is discarded from the original signal. Similarly, for PLI cancellation, harmonics of contributing segments are computed to reduce the interference. Cuomo, Farina, and Piccialli [42] suggested that some "a priori" information about signal components can be helpful for improving the performance of signal denoising. Thus, authors developed recursive filtering $(\mathrm{RF})$ based scheme by using a probabilistic Bayesian framework.

According to Hesar and Mohebbi [43], the ECG signals are non-linear in nature which is the main issue that affects the filtering process. Moreover, the noise contamination also affects the ECG signal analysis performance. In order to overcome these issues, Kalman filtering based approaches have been developed but due to recursion problem these techniques need to be improved for better analysis.

Based on these studies, a comparative study is carried out as presented in table 1. This study shows that several approaches are introduced for ECG signal filtering but very less amount of work is carried out which can be robust in terms of linear and non-linear systems. Moreover, the 
complexity and reconstruction quality is also challenging task which can degrade the performance. Hence, there is a scope to improve the performance of ECG signal denoising. Thus, this work presented a hybrid approach using extended kalman filter with linearization, wavelet transform and adaptive thresholding.

\section{Proposed APPROACH}

This section presents the proposed approach for ECG signal filtering using adaptive techniques of signal filtering. The proposed approach is a combination of Kalman filter and Wavelet transformation technique. The complete process of proposed approach is depicted in figure 1 .

During ECG signal acquisition, the signal gets contaminated due to different types of noise such as synthetic noise, electrode noise, motion artifact and baseline drifts. A brief discussion about noise is presented in section III A.

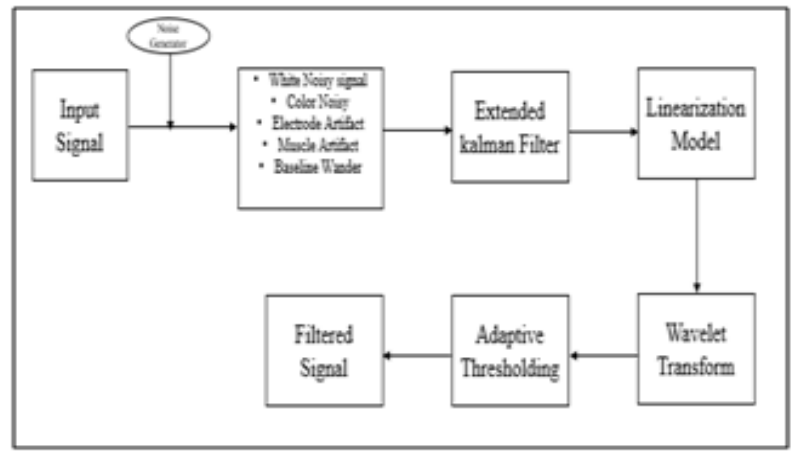

Fig. 1. Block diagram of Proposed approach (LKFWAT)

\section{A. ECG signal Noise model}

This section presents a brief model about different type of noises.

- Synthetic Noise

During processing the ECG signals, synthetic noise can be added into the original signal in the form of white noise or color noise. The white noise is generated as a random signal with a power spectral density. Similarly the color synthetic noise also can be generated which has low -frequency and this noise is inversely proportional to the power spectral density. In ECG signal acquisition, the slight fluctuations in the resistance can generate flicker noise. In this work, a spectral density function is considered, and the variation is slope can be used for modeling the color noise as $S(f) \propto \frac{\sigma^{2}}{f^{\tau}}$ where $S$ is the generated signal, $f$ denotes the frequency value, $\sigma$ is the variance of the signal, and $\tau$ denotes the slope variation parameter. In this model, if $\tau=0$ then the white noise is generated, and $\tau=1$ generates the flicker noise.

- Noise generated due to electrode contact

During signal acquisition, contact between electrodes and skin get disconnected thus an interference occurs which is known as electrode contact noise. This noise can be generated due to vibration. This loss in contact can be temporary or permanent where frequent disconnection occurs. This frequent switching can cause large artifacts.

- Motion Artifact

This is type of transient base line change which is caused due to variation between electrodes -skin impedance and electrode motion. During acquisition, the variation in the ECG signal impedance formulates a voltage divider with the amplifier input impedance. Generally, the motion artifact is caused due to the vibrations or movements of subjects.

- Baseline drift

This is a short term variation in the original signal where original signal is slightly deviated from the original signal due to electrical fluctuations, temperature fluctuations, lamp instability and several other factors.

\section{B. Kalman Filter, Extended Kalman filter and proposed Kalman filter}

In this sub-section a brief discussion about existing Kalman filtering technique and its implementation for signal filtering, extended Kalman filtering and its implementation for signal denoising and proposed Modified extended Kalman filter are discussed.

- Kalman Filter

Kalman filter is a recursive data filtering technique widely adopted technique for data filtering and data pre-processing. A general architecture of Kalman filtering technique is depicted in figure 2 .

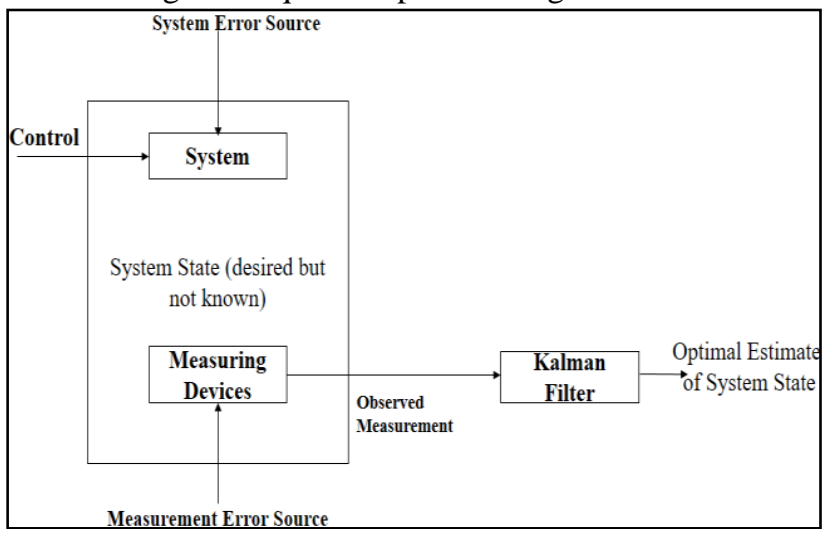

Fig.2. Kalman filtering architecture

The main aim of this approach is to identify the system states which contain random error in the data. Dynamic equation and with measurement noise equation is shown in equation 1 .

$x_{k+1}=A x_{k}+u_{k}$ and

$z_{k}=H x_{k}+v_{k}$

$v_{k}$ denotes the white Gaussian noise

$v_{k}$ is called white Gaussian noise which is not correlated with any other random 
variables. Kalman filter is applied in two steps: the time update step and the measurement update step. Figure 3 shows these steps.

\begin{tabular}{|c|c|}
\hline $\begin{array}{l}\text { Predictor Phase } \\
\text { 1. Project the state ahead } \\
\quad \hat{x}_{k}^{-}=A \hat{x}_{k-1}^{-}+B u(k) \\
\text { 2. Project the error covariance } \\
\text { ahead } \\
\qquad P_{k}^{-}=A P_{k-1} A^{T}+Q\end{array}$ & $\begin{array}{l}\text { Update Phase } \\
\text {.Compute the Kalman Gain } \\
K_{k}=P_{k}^{-} H^{T}\left(H P_{k}^{-} H^{T}+R\right)^{-1} \\
\text { 2. Update Estimate with } \\
\text { measurement } \\
x_{k}=\hat{x}_{k}^{-}+K_{k}\left(z_{k}-H \hat{x}_{k}^{-}\right) \\
\text {3. Update the error covariance } \\
P_{k}=\left(I-K_{k} H\right) P_{k}^{-}\end{array}$ \\
\hline
\end{tabular}

Fig.3. Kalman filtering approach

- Extended Kalman filter

The convolution models of Kalman filter are only applicable for linear problems but generally the real-world problems are based on the non-linear nature. Thus, the current Kalman filters need to extend so that it can handle non-linear dynamic models. Hence, extended Kalman filter based scheme is developed [44, 9]. Let $\mathrm{D}$ be a discrete non-linear system is expressed as $x_{k+1}=f\left(x_{k}, w_{k}\right)$ and the observation of this input system is a linear approximation given as $y_{k}=g\left(x_{k}, v_{k}\right)$. This linear approximation is close to the reference point $\left(\hat{x}_{k}, \widehat{w}_{k}, \hat{v}_{k}\right)$ which can be expressed as:

$\left\{\begin{array}{c}x_{k+1} \approx f\left(\hat{x}_{k}, \widehat{w}_{k}\right)+\mathbb{A}_{k}\left(x_{k}-\hat{x}_{k}\right)+\mathbb{F}_{k}\left(w_{k}-\widehat{w}_{k}\right) \\ y_{k} \approx g\left(\hat{x}_{k}, \hat{v}_{k}\right)+\mathbb{C}_{k}\left(x_{k}-\hat{x}_{k}\right)+\mathbb{G}_{k}\left(v_{k}-\hat{v}_{k}\right)\end{array}\right.$

Where, $x_{k}$ represents the state vector and $y_{k}$ represents the observation vector. $\mathbb{A}_{k}, \mathbb{F}_{k}, \mathbb{C}_{k}$ and $\mathbb{G}_{k}$ denotes the Jacobian matrices which are given as follows:

$$
\left\{\begin{array}{cc}
\mathbb{A}_{k}=\left.\frac{\partial f\left(x, \widehat{w}_{k}\right)}{\partial x}\right|_{x_{k}=\hat{x}_{k}} \quad \mathbb{F}_{k}=\left.\frac{\partial f\left(\hat{x}_{k}, w_{k}\right)}{\partial w}\right|_{w_{k}=\widehat{w}_{k}} \\
\mathbb{C}_{k}=\left.\frac{\partial g\left(x, \hat{v}_{k}\right)}{\partial x}\right|_{x_{k}=\hat{x}_{k}} \quad \mathbb{G}_{k}=\left.\frac{\partial g\left(\hat{x}_{k}, v\right)}{\partial v}\right|_{v=\hat{v}_{k}}
\end{array}\right.
$$

$f($.$) Denotes the nonlinear process and g($. denotes the observation vector functions, $v_{k}$ measurement noise with a covariance matrix as $R_{k}=E\left\{v_{k} v_{k}^{T}\right\}, w_{k}$ represents state noise with a covariance matrix as $Q_{k}=E\left\{w_{k} w_{k}^{T}\right\}$. With the help of these functions, the EKF equation can be expressed as:

$$
\left\{\begin{array}{c}
\hat{x}_{k / k-1}=f\left(\hat{x}_{k / k-1}, 0\right) \\
P_{k / k-1}=\mathbb{A}_{k} P_{k-1 / k-1} \mathbb{A}_{k}^{T}+\mathbb{F}_{k} Q_{k} \mathbb{F}_{k}^{T} \\
\hat{x}_{k / k}=\hat{x}_{k / k-1}+K_{k}\left[y_{k}-g\left(\hat{x}_{k / k-1}, 0\right)\right] \\
K_{k}=P_{k / k-1} C_{k}^{T}\left[C_{k} P_{k / k-1} \mathbb{C}_{k}^{T}+\mathbb{G}_{k}^{T}\right]^{-1} \\
P_{k / k}=P_{k / k-1}-K_{k} C_{k} P_{k / k-1}
\end{array}\right.
$$

In Eq. (3), as discussed before the state vector and observation can be represented as $\hat{x}_{k / k-1}=$ $\mathbb{E}\left\{x_{k} \mid y_{k-1}, y_{k-2}, \ldots, y_{1}\right\} \quad$ represents estimated state vector estimated at $k$ for $y_{1}$ to $y_{k-1}$ observations, similarly,

$\hat{x}_{k / k-1}=\mathbb{E}\left\{x_{k} \mid y_{k}, y_{k-1}, \ldots, y_{1}\right\}$ also denotes the state vector at time $k$ for $y_{1}$ to $y_{k}$ number of observations. This model provides the final state vector and observations. In order to further improve the signal, linearization model is applied which helps to convert the non-linear model to the linear model. Generally, these signals are nonlinear in nature which causes complexity in signal analysis. In order to perform the appropriate signal analysis, signal need to be transformed in a linear structure which can make interpretation easier. With the help of this, a new model for extended Kalman filter is developed which is called as Linearized Extended Kalman Filter (LEKF). This linearization model for EKF helps to improve the observation of EKF. This is performed based on the state variables as $x, w$ and $v$ which can be expressed as:

$\left\{\begin{array}{l}\hat{x}=\mathbb{F}\left(\hat{x}_{k}, \widehat{w}_{k}, \hat{v}_{k}\right) \\ \widehat{w}=\mathbb{G}\left(\hat{x}_{k}, \widehat{w}_{k}, \hat{v}_{k}\right) \\ \hat{v}=\mathbb{A}\left(\hat{x}_{k}, \widehat{w}_{k}, \hat{v}_{k}\right)\end{array}\right.$

Where $\frac{\partial \mathbb{F}}{\partial x}=\frac{-2 x^{2}-w^{2}}{\sqrt{x^{2}+y^{2}}}+1, \frac{\partial \mathbb{G}}{\partial x}=\frac{-2 x^{2}-w^{2}}{\sqrt{x^{2}+y^{2}}}+$ $1, \frac{\partial \mathbb{F}}{\partial y}=\frac{-x y}{\sqrt{x^{2}+y^{2}}}-\omega$ and $\frac{\partial \mathbb{G}}{\partial y}=\frac{-2 y^{2}-x^{2}}{\sqrt{x^{2}+y^{2}}}+1$

\section{Wavelet Transform and adaptive thresholding for} Signal Denoising

This subsection describes the implementation of wavelet transform for signal denoising. The wavelet transform of the given input signal is a scaled and shifted form of the original input pattern hence it is called as "mother wavelet". The inner product of the observed signal $(t)$, shifted and mother wavelet is performed which generates the continuous wavelet transform.

This wavelet transform can be expressed as: 
$\left\langle f(t), \psi_{a, s}\right\rangle=W T_{f}(s, \tau)=$

$\frac{1}{\sqrt{s}} \int f(t) \psi^{*}\left(\frac{t-\tau}{s}\right) d t$

Where, $(s>0)$ represents the scale variable and $\tau$ represents the time shift variable. With the help of this model the discrete wavelet transform model can be presented which is the redundant representation of (4). The discrete representation of continuous wavelet can be obtained by setting the parameter $s=s_{0}^{i}$ and $\tau=k \tau_{0} s_{0}^{i}$ where $i$ and $k$ are integer values and $s_{0}>1$, in this work, the values of $S_{0}$ and $\tau_{0}$ are considered as 2 and 1 respectively, which can be used for representing the parameters as $s=2^{i}$ and $\tau=k 2^{i}$ which denotes the dyadic wavelet transform which can be expressed as:

$\psi_{i, k}=2^{-\frac{i}{2}} \psi\left(2^{-1} t-k\right)$

Thus, the DWT decomposition for any given signal $f(t)$ can be given as:

$$
\begin{aligned}
& f(t)=\sum_{k=-\infty}^{\infty} C_{N, K \varphi}\left(2^{-N} t-k\right)+ \\
& j=10 k=\infty \infty j, k 2-j 2 \psi 2-j t-k
\end{aligned}
$$

The approximation coefficients for $N$ level DWT is given by $C_{N, K}$ and detailed coefficient is represented by $d, \psi$ is the wavelet function and $\varphi$ is a scaling function. Based on the filter bank theory and multi-resolution analysis, the above given signal in (6) can be represented as:

$f(t)=$

$\sum_{k \in Z} \mathcal{C}_{L}(k) \varphi_{L, k}(t)+\sum_{j=1}^{L} d_{j}(k) \psi_{j, k}(t)$

Where detailed coefficients and approximation coefficients can be re-written as:

$$
\begin{aligned}
& \mathcal{C}_{L}(n)=\left\langle f, \varphi_{j, n}\right\rangle=\frac{1}{\sqrt{2}} \sum_{k} h(2 n- \\
& k C L-1 n d j n=f, \quad \psi j, n=12 k g 2 n-k s j-1 n
\end{aligned}
$$

$\varphi(t)$ denotes the scaling function which satisfies the condition as $\int \varphi(t) d t=1, h(n)$ represents the low-pass filter and $g(n)$ denotes the high-pass filter. Hence, the complete DWT is formulated using dyadic scales and an orthonormal basis is formed to perform the signal analysis.

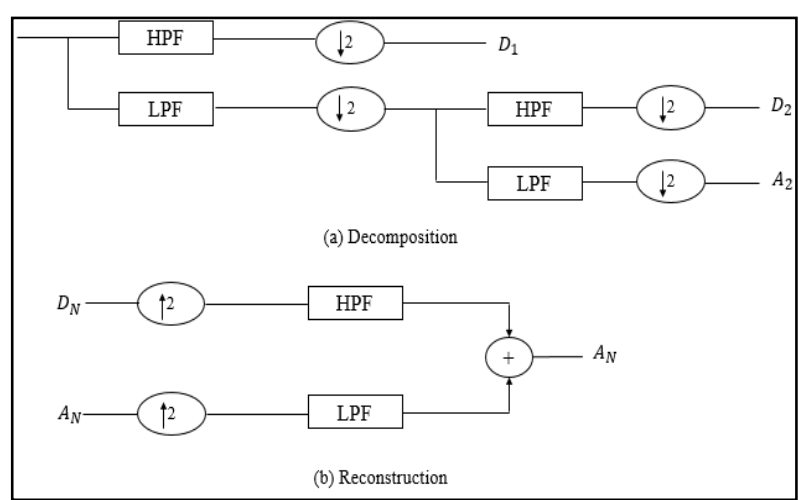

Fig.4.Wavelet transforms (a) wavelet signal decomposition and (b) decomposed signal reconstruction

With the help of DWT process, the signal is decomposed into filter pairs as low pass filter (LPF) and high pass filter (HPF) and the cut-off frequency is the middle frequency of the input signal. 2-level wavelet-based signal decomposition is depicted in the figure 4 which shows two detailed coefficients and two approximation coefficients. In order to compute the detailed and approximated coefficients as $d_{j}(n)$ and $\mathcal{C}_{L}(n)$, the coefficients of high pass and low pass filter are required which can be obtained using Symlet wavelet transform.

- Adaptive thresholding

After decomposing the signal, low pass filtered signal data is achieved. However, due to different types of noises and noise with high $\mathrm{dB}$, the wavelet-based approach suffers from the denoising performance. Hence, thresholding methods for signal denoising are considered which are generally classified as: (a) soft-thresholding and (b) Hard-thresholding [45]. Due to increased complexity and inappropriate parameter selection, the existing techniques suffer from the selection of thresholding process. In order to overcome this issue, an adaptive thresholding process is incorporated.

According to the adaptive threshold scheme, each sub-band of decomposed signal is considered where Generalized Gaussian Distribution (GGD) is computed to estimate the signal threshold. The GGD can be computed as:

$G D_{\sigma_{X}, \gamma}(x)=$

$\mathcal{C}\left(\sigma_{X}, \gamma\right) \exp -\left[\mu\left(\sigma_{X}, \gamma\right)|x|\right]^{\gamma}$

Where $\gamma>0$ and $-\infty<x<\infty, \mu\left(\sigma_{X}, \gamma\right)=$ $\sigma_{X}^{-1}\left[\frac{\left(\frac{3}{\gamma}\right) \zeta}{\left(\frac{1}{\gamma}\right) \zeta}\right], \mathcal{C}\left(\sigma_{X}, \gamma\right)=\frac{\gamma \cdot \mu\left(\sigma_{X}, \gamma\right)}{2 \zeta(1 / \gamma)}$

Where $\zeta=\int_{0}^{\infty} e^{-u} u^{(t-1)} d u$, here $\gamma$ is a constant which is varying from 0.5 to $1, \sigma_{X}$ is the standard deviation of the signal. In this process, each sub-band compute $\sigma_{\mathrm{X}}$ and $\gamma$ values to compute the threshold such that it minimizes Bayesian risk and 
gives the optimal threshold. The expected mean square error in the Bayesian form can be given as:

$\phi(T)=E(\hat{X}-X)^{2}=E_{X} E_{Y \mid X}(\hat{X}-X)^{2}$

Where $\widehat{X}=\eta T, Y \mid X, \eta$ denotes the soft-threshold estimator. The optimal threshold $T^{*}$ is expressed as:

$T^{*}\left(\sigma_{x}, \gamma\right)=\arg \min _{T} \phi(T)$

With the help of this the, observed threshold is given as $T_{B}\left(\sigma_{x}\right)=\frac{\sigma^{2}}{\sigma_{X}}$ and the normalized threshold is given as $\frac{T_{B}}{\sigma}$. This shows that the normalized threshold is inversely proportional to the standard deviation of the signal and proportional to the standard deviation of noise. Based on the obtained threshold, rule for noise reduction is defined which are as follows:

-If $\frac{\sigma}{\sigma_{X}} \ll 1$ then the signal is considered as stronger that the noise hence the value of $\frac{T_{B}}{\sigma}$ is selected very small which helps to preserve the signal.

- If $\frac{\sigma}{\sigma_{X}} \gg 1$ then the presence of noise is considered more in the original signal hence $\frac{T_{B}}{\sigma}$ is selected larger to remove the noise.

\section{RESULTS AND DISCUSSION}

This section presents experimental study using proposed approach of signal filtering. This experimental analysis is conducted by using publicly available arrhythmia dataset MIT-BIH [46].

\section{A. Dataset details}

The proposed approach is implemented using publically available ECG data which is known as Physionet MIT-BIH arrhythmia database. This dataset contains total 48 ECG samples where each sample is digitized at 360 samples per second per channel. This dataset is stored into three types of files which are text header file(. hea), Reference annotation file(.atr), and Digitized binary file(.dat). The header files contains all information related to ECG signals such as format of ECG signal, number of samples, ECG leads, history of patient and other clinical information. Figure 5 (a) shows a sample ECG signal used in this work for signal filtering approach.

\section{B. Performance measurement metrics}

The performance of ECG signal denoising is measured with the help of various parameters. These parameters are as follows:

(1) Mean Squared Error: $M S E=\frac{1}{N} \sum_{i=1}^{i-N}(X(i)-$ Yi2

(2) Root Mean Square Error: $R M S E=$ $\sqrt{\frac{1}{N} \sum_{i=1}^{i-N}(X(i)-Y(i))^{2}}$

(3) Peak Signal to noise ratio: $P S N R=$ $10 \log 10\left(\frac{\sum_{i=1}^{N} M A X^{2}}{M S E}\right)$

(4) The percent root mean square difference: $P R D=$ $100 \times \sqrt{\frac{\sum_{i=1}^{i-N}(Y(i)-X(i))^{2}}{\sum_{i=1}^{N}(X(i))^{2}}}$

(5) Cross correlation

\section{Comparative performance}

This section presents a comparative analysis of denoising performance of proposed approach and existing approaches. First of all, the complete process of signal filtering using proposed approach is presented and later existing works is considered to compare the performance.

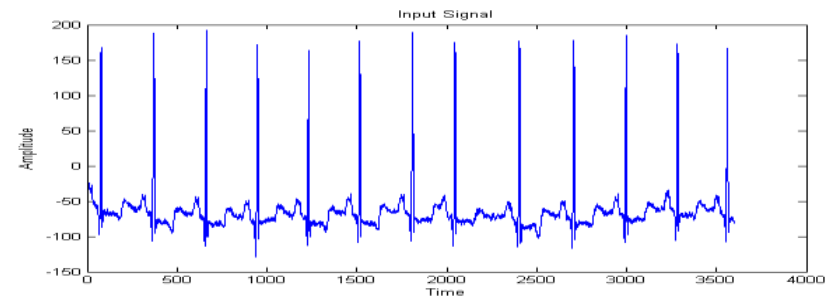

Fig. 5. (a). Input Signal 


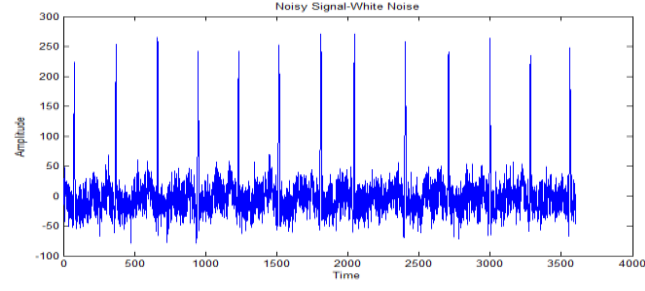

Fig. 5.(b) White noise ECG 5dB

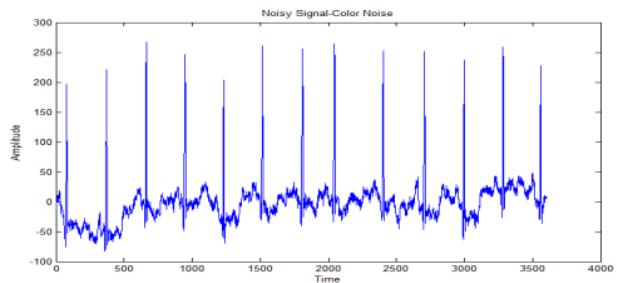

Fig. 5(d) Color noise added ECG 5dB

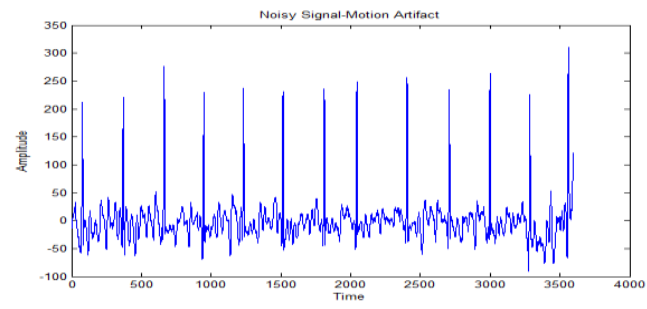

Fig. 5(f) Motion artifact Added ECG 5dB

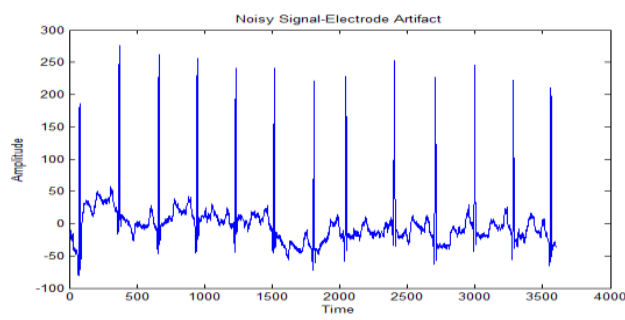

Fig. 5(h) Electrode artifact 5db noise

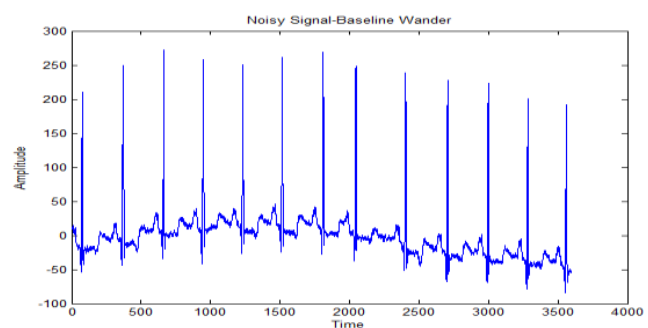

Fig. 5(j) baseline wander added 5dB

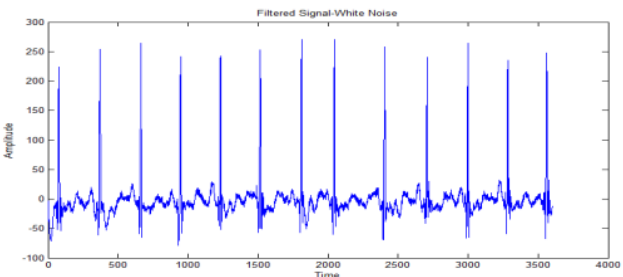

Fig. 5.(c) Filtered White Noise ECG

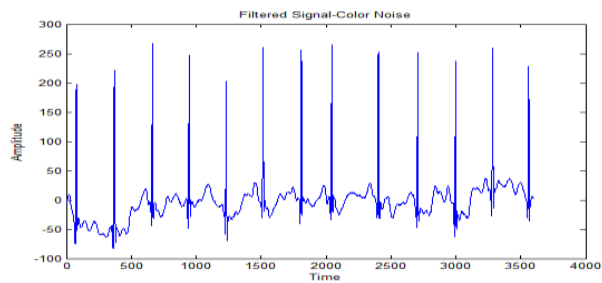

Fig. 5 (e) Filtered Color Noise

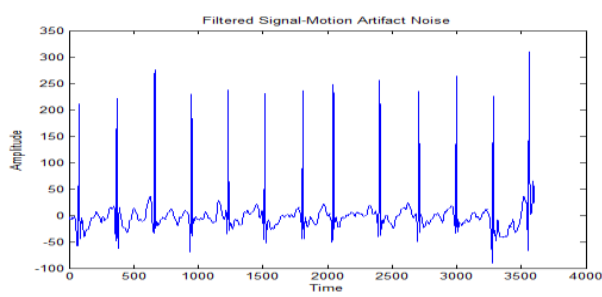

Fig. 5(g) Filtered Motion Artifact

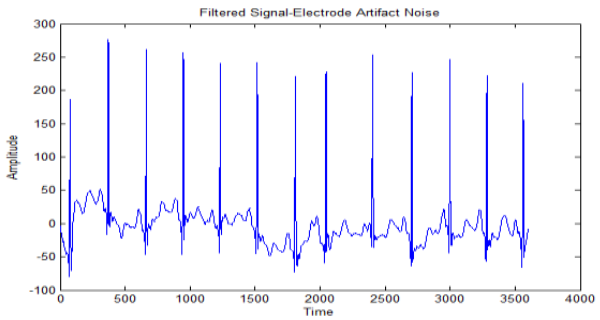

Fig. 5(i) Filtered Electrode Artifact

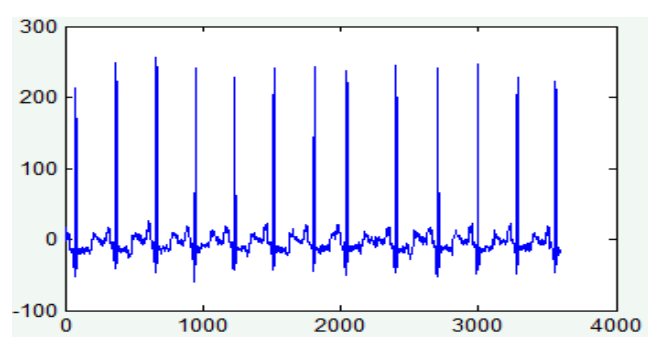

Fig. 5 (k) Baseline wander filtered ECG

Fig .5. Complete process of proposed ECG denoising approach 
Table 2.Signal denoising performance for 5 and $10 \mathrm{~dB}$ noise for "101.mat" signal in table 2 .

\begin{tabular}{|c|c|c|c|c|c|c|}
\hline Noise dB & Quantitative Metrics & White Noise & Color Noise & $\begin{array}{c}\text { Motion } \\
\text { Artifact }\end{array}$ & $\begin{array}{c}\text { Electrode } \\
\text { Artifact }\end{array}$ & $\begin{array}{c}\text { Baseline } \\
\text { Wander }\end{array}$ \\
\hline \multirow{4}{*}{$5 \mathrm{~dB}$} & PSNR & 46.53 & 33.99 & 41.93 & 47.92 & 42.28 \\
\cline { 2 - 7 } & MSE & 1.46 & 1.0264 & 4.04 & 1.0016 & 3.65 \\
\cline { 2 - 7 } & MAE & 0.9394 & 0.801 & 1.51 & 0.78 & 1.51 \\
\cline { 2 - 7 } & RMSE & 1.20 & 1 & 2 & 1 & 1.91 \\
\cline { 2 - 7 } & CC & 0.99 & 0.99 & 0.99 & 0.99 & 0.99 \\
\hline \multirow{4}{*}{$10 \mathrm{~dB}$} & PSNR & 48.15 & 47.43 & 41.92 & 47.31 & 42.80 \\
\cline { 2 - 7 } & MSE & 1.01 & 1.178 & 4.15 & 1.192 & 3.34 \\
\cline { 2 - 7 } & MAE & 0.75 & 0.857 & 1.54 & 0.854 & 1.44 \\
\cline { 2 - 7 } & RMSE & 1 & 11.09 & 1.24 & 1.09 & 1.82 \\
\cline { 2 - 7 } & CC & 0.92 & 0.99 & 0.998 & 0.99 & 0.998 \\
\hline
\end{tabular}

The above given figure 5 shows complete process of proposed approach where input signal is considered and different types of noises are considered such as white noise, color noise, electrode artifact, motion artifact and baseline wander which are added to the original input signal. The original signal is depicted in $5(\mathrm{a})$, different types of noisy signal such as white noise, color noise, motion artifact, electrode artifact and baseline wander are depicted in figure 5(b)-5(f). Signal filtering is performed using proposed approach and corresponding filtered signals are depicted in figure $5(\mathrm{~g})-5(\mathrm{k})$. Based on these filtered signals, the performance of proposed approach is measured as presented
The table 2 shows a performance analysis using proposed approach where the noise is varied as $5 \mathrm{~dB}$ and $10 \mathrm{~dB}$ by considering white noise, Color Noise, Motion Artifact, Electrode Artifact and Baseline Wander. The performance of proposed approach is compared in terms of aforementioned metrics. In this study, for $5 \mathrm{~dB}$ PSNR the maximum PSNR is obtained as $47.92 \mathrm{~dB}$ for Electrode Artifact and similarly, for $10 \mathrm{~dB}$ highest PSNR is obtained as $48.15 \mathrm{~dB}$ for white noise. In next stage, the performance of proposed approach is compared with existing techniques to show the robust performance of proposed denoising method. In order to perform the signal denoising, several samples of ECG are considered which are obtained from MIT-BIH dataset.

Table 3. SNR performance for Muscle Artifact removal for $1.25 \mathrm{~dB}$ noise.

\begin{tabular}{|c|c|c|c|c|c|}
\hline \multirow{2}{*}{$\begin{array}{c}\text { Sample } \\
\text { number }\end{array}$} & $\begin{array}{c}\text { S-Transform } \\
{[48]}\end{array}$ & $\begin{array}{c}\text { BPNN } \\
{[22]}\end{array}$ & $\begin{array}{c}\text { WT-Sub-band } \\
{[47]}\end{array}$ & $\begin{array}{c}\text { Butterworth } \\
\text { Guided Filter } \\
{[49]}\end{array}$ & $\begin{array}{c}\text { Proposed } \\
\text { approach }\end{array}$ \\
\hline 1 & 10.89 & 8.55 & 2.82 & 11.93 & 14.30 \\
\hline 2 & 10.42 & 8.30 & 2.9 & 10.22 & 13.05 \\
\hline 3 & 8.66 & 8.64 & 2.7 & 8.57 & 11.80 \\
\hline 4 & 8.51 & 8.49 & 2.76 & 11.29 & 14.20 \\
\hline 5 & 9.67 & 9.69 & 2.86 & 14.19 & 16.30 \\
\hline 6 & 8.61 & 8.12 & 2.95 & 11.2 & 15.31 \\
\hline 7 & 9.67 & 8.31 & 2.58 & 11.19 & 14.30 \\
\hline 8 & 10.61 & 8.60 & 2.85 & 11.54 & 13.80 \\
\hline 9 & 10.56 & 8.02 & 2.92 & 12.1 & 15.30 \\
\hline 10 & 9.01 & 8.01 & 2.87 & 10.71 & 13.50 \\
\hline
\end{tabular}

The performance of proposed approach is compared with existing techniques such as WT-Sub-band [47], S-Transform [48], BPNN [22], and Butterworth guided filter [49]. First of all, the performance is measured for muscle artifact noise in terms of SNR for varied noise $\mathrm{dB}$ as $1.25 \mathrm{~dB}$ and $5 \mathrm{~dB}$ in table 3 and table 4 respectively. Similarly, the performance for $5 \mathrm{~dB}$ noise of muscle artifact is measured. The measured performance is presented in table 4 . 
Table 4. SNR performance for Muscle Artifact removal for $5 \mathrm{~dB}$ noise

\begin{tabular}{|c|c|c|c|c|c|}
\hline \multirow{2}{*}{$\begin{array}{c}\text { Sample } \\
\text { number }\end{array}$} & $\begin{array}{c}\text { S-Transform } \\
{[48]}\end{array}$ & $\begin{array}{c}\text { BPNN } \\
{[22]}\end{array}$ & $\begin{array}{c}\text { WT-Sub-band } \\
{[47]}\end{array}$ & $\begin{array}{c}\text { Butterworth Guided } \\
\text { Filter } \\
{[49]}\end{array}$ & $\begin{array}{c}\text { Proposed } \\
\text { approach }\end{array}$ \\
\hline 1 & 12.63 & 8.21 & 6.4 & 12.45 & 16.20 \\
\hline 2 & 12.76 & 8.39 & 6.49 & 12.68 & 15.88 \\
\hline 3 & 9.94 & 8.40 & 6.17 & 9.06 & 14.22 \\
\hline 4 & 9.75 & 8.65 & 6.29 & 12.68 & 13.61 \\
\hline 5 & 11.69 & 8.14 & 6.49 & 16.53 & 17.20 \\
\hline 6 & 9.91 & 9.04 & 6.54 & 11.77 & 14.29 \\
\hline 7 & 12.53 & 8.84 & 6.02 & 12.79 & 14.28 \\
\hline 8 & 12.89 & 8.63 & 6.44 & 12.05 & 14.09 \\
\hline 9 & 13.44 & 8.86 & 6.56 & 12.88 & 13.55 \\
\hline 10 & 10.15 & 8.46 & 6.49 & 13.58 & 15.60 \\
\hline
\end{tabular}

The above given table shows a comparative performance for $5 \mathrm{~dB}$ muscle artifact noise denoising comparative performance. This comparative study shows that the

Similarly, the performance of proposed approach is measured in terms of RMSE for varied noise as 1.25 and $5 \mathrm{~dB}$. This proposed approach achieves better performance in terms of comparative performance is given in table 5 .

SNR.

Table 5. RMSE performance for Muscle Artifact removal for $1.25 \mathrm{~dB}$ noise

\begin{tabular}{|c|c|c|c|c|c|}
\hline \multirow{2}{*}{$\begin{array}{c}\text { Sample } \\
\text { number }\end{array}$} & $\begin{array}{c}\text { S-Transform } \\
{[48]}\end{array}$ & $\begin{array}{c}\text { BPNN } \\
{[22]}\end{array}$ & $\begin{array}{c}\text { WT-Sub-band } \\
\text { [47] }\end{array}$ & $\begin{array}{c}\text { Butterworth Guided } \\
\text { Filter } \\
{[49]}\end{array}$ & $\begin{array}{c}\text { Proposed } \\
\text { approach }\end{array}$ \\
\hline 1 & 0.4 & 0.6 & 0.8 & 0.3 & 0.12 \\
\hline 2 & 0.42 & 0.55 & 0.82 & 0.28 & 0.16 \\
\hline 3 & 0.46 & 0.55 & 0.83 & 0.36 & 0.22 \\
\hline 4 & 0.42 & 0.62 & 0.76 & 0.38 & 0.26 \\
\hline 5 & 0.41 & 0.60 & 0.81 & 0.25 & 0.21 \\
\hline 6 & 0.43 & 0.56 & 0.83 & 0.36 & 0.23 \\
\hline 7 & 0.39 & 0.59 & 0.85 & 0.38 & 0.29 \\
\hline 8 & 0.38 & 0.61 & 0.80 & 0.39 & 0.28 \\
\hline 9 & 0.39 & 0.56 & 0.82 & 0.35 & 0.31 \\
\hline 10 & 0.4 & 0.6 & 0.81 & 0.39 & 0.29 \\
\hline
\end{tabular}


Table 6. RMSE performance for Muscle Artifact removal for 5 dB noise

\begin{tabular}{|c|c|c|c|c|c|}
\hline \multirow{2}{*}{$\begin{array}{l}\text { Sample } \\
\text { number }\end{array}$} & \multicolumn{5}{|c|}{$5 \mathrm{~dB}$} \\
\hline & S-Transform [48] & $\begin{array}{c}\text { BPNN } \\
{[22]}\end{array}$ & WT-Sub-band [47] & $\begin{array}{c}\text { Butterworth Guided Filter } \\
\text { [49] }\end{array}$ & Proposed approach \\
\hline 1 & 0.26 & 0.41 & 0.5 & 0.25 & 0.21 \\
\hline 2 & 0.27 & 0.4 & 0.51 & 0.24 & 0.18 \\
\hline 3 & 0.3 & 0.43 & 0.52 & 0.3 & 0.24 \\
\hline 4 & 0.32 & 0.44 & 0.55 & 0.28 & 0.26 \\
\hline 5 & 0.3 & 0.41 & 0.51 & 0.21 & 0.15 \\
\hline 6 & 0.31 & 0.44 & 0.56 & 0.24 & 0.16 \\
\hline 7 & 0.28 & 0.46 & 0.58 & 0.25 & 0.22 \\
\hline 8 & 0.26 & 0.41 & .0 .56 & 0.26 & 0.22 \\
\hline 9 & 0.29 & 0.45 & 0.55 & 0.24 & 0.21 \\
\hline 10 & 0.3 & 0.41 & 0.55 & 0.25 & 0.2 \\
\hline
\end{tabular}

The complete experimental study shows that the proposed approach achieves better performance in terms of SNR for muscle artifact noise scenario.

\section{CONCLUSION}

This study is focused on removing the noise such as electrode movement, muscle movement and other types of interference from ECG signal fusing bio-medical signal processing approach. These conventional approaches are based on both linear and non-linear methods where developing robust approach is a challenging task and also these systems suffer from the poor performance of error estimation. Moreover, noise reduction and signal reconstruction quality are also serious issues in state-of-art techniques. To overcome these issues, a novel hybrid approach is developed where error estimation, signal linearization, and wavelet decomposition are performed to reconstruct the better quality signal. A Simulation study is carried out and the obtained performance is compared with the existing approaches which show a significant improvement in the signal quality using the proposed approach. These high qualities filtered ECG signals can be used for the identification and classification of diseases with greater accuracy and in future work, these signals will be taken into account.

\section{APPENDIX}

The proposed approach is implemented using publically available ECG data which is known as Physionet MIT-BIH arrhythmia database. In order to perform the signal denoising, several samples of ECG are considered which are used in Table.3, Table.4, Table.5 and Table.6. Those sample numbers related filenames are shown in below.

1. Sample number 1 is extracted from 103.mat signal file.

2. Sample number 2 is extracted from 105.mat signal file.

3. Sample number 3 is extracted from 111.mat signal file.

4. Sample number 4 is extracted from 116.mat signal file.

5. Sample number 5 is extracted from 122.mat signal file.

6. Sample number 6 is extracted from 205.mat signal file.

7. Sample number 7 is extracted from 213.mat signal file.

8. Sample number 8 is extracted from 219.mat signal file.

9. Sample number 9 is extracted from 223.mat signal file.

10. Sample number 10 is extracted from 230.mat signal file.

\section{REFERENCES}

1. The top 10 causes of death (2018) [Online] Available: https://www.who.int/news-room/fact-sheets/detail/the-top-10-causesof-death (accessed 5 February 2019)

2. J. Mackay, G. Mensah, S. Mendis, and K. Greenlund, (2004, Mar, 8), The atlas of heart disease and stroke, World Health Organization, [Online], Vol. $\quad 5, \quad$ Available: https://apps.who.int/iris/handle/10665/43007

3. H. H. Chang, and J. M. Moura, "Biomedical signal processing" in Biomedical engineering and design handbook, 2nd edition, Myer kutz, McGraw Hill, 2010, pp.559-579.

4. M. Blanco-Velasco, B. Weng, and K. E. Barner, "ECG signal denoising and baseline wander correction based on the empirical mode decomposition", in Computers in biology and medicine, Vol.38 No.1, pp.1-13, 2008.

5. K.M. Chang, "Arrhythmia ECG noise reduction by ensemble empirical mode decomposition", in Sensors, Vol.10 No.6, pp.6063-6080, 2010.

6. Z. Zhao, and J. Liu, "Baseline wander removal of ECG signals using empirical mode decomposition and adaptive filter" in 4th International Conference on Bioinformatics and Biomedical Engineering, IEEE, pp. 1-3, Jun 2010.

7. Y. D. Lin, and Y. H. Hu, "Power-line interference detection and suppression in ECG signal processing" in IEEE Transactions on Biomedical Engineering, Vol.55 No.1, pp.354-357, 2008

8. M. Niknazar, B. Rivet, and C. Jutten, "Fetal ECG extraction by extended state Kalman filtering based on single-channel recordings", in IEEE Transactions on Biomedical Engineering, Vol.60 No.5, pp.1345-1352, 2013.

9. O. Sayadi, and M. B. Shamsollahi, "ECG denoising and compression using a modified extended Kalman filter structure" IEEE Transactions on Biomedical Engineering, Vol.55 No.9, pp.2240-2248, 2008.

10. J. Gao, H. Sultan, J. Hu, and W. W. Tung, "Denoising nonlinear time series by adaptive filtering and wavelet shrinkage: a comparison", in IEEE signal processing letters, Vol.17 No.3, pp.237-240, 2010.

11. H. Y. Lin, S. Y. Liang, Y. L. Ho, Y. H. Lin, and H. P. Ma, "Discrete-wavelet-transform-based noise removal and feature extraction for ECG signals" in Innovation and Research in BioMedical engineering (Irbm), Vol.35 No.6, pp.351-361, 2014.

12. L. N. Sharma, S. Dandapat, and A. Mahanta, "ECG signal denoising using higher order statistics in Wavelet subbands" in Biomedical Signal Processing and Control, Vol.5 No.3, pp.214-222, 2010.

13. S. A. Taouli, and F. Bereksi-Reguig, "Noise and baseline wandering suppression of ECG signals by morphological filter", in Journal of medical engineering \& technology, Vol.34 No.2, pp.87-96, 2010

14. R. Vullings, B. De Vries, and J. W. Bergmans, "An adaptive Kalman filter for ECG signal enhancement", in IEEE transactions on biomedical engineering, Vol.58 No.4, pp.1094-1103, 2011.

15. K. M. Chang, and S. H. Liu, "Gaussian noise filtering from ECG by Wiener filter and ensemble empirical mode decomposition", in Journal of Signal Processing Systems, Vol.64 No.2, pp.249-264, 2011.

16. E. S. A. El-Dahshan, "Genetic algorithm and wavelet hybrid scheme for ECG signal denoising", in Telecommunication Systems, Vol.46 No.3, pp.209-215, 2011

17. M. A. Kabir, and C. Shahnaz, "Denoising of ECG signals based on noise reduction algorithms in EMD and wavelet domains" in Biomedical Signal Processing and Control, Vol.7 No.5, pp.481-489, 2012. 
18. S. Poungponsri, and X. H. Yu, "An adaptive filtering approach for electrocardiogram (ECG) signal noise reduction using neural networks" in Neurocomputing, Vol.117, pp.206-213, 2013

19. S Sahoo, B. Kanungo, S. Behera, and S. Sabut, "Multiresolution wavelet transform based feature extraction and ECG classification to detect cardiac abnormalities" Measurement, 108, pp.55-66, 2017.

20. S. Banerjee, and M. Mitra, "Application of cross wavelet transform for ECG pattern analysis and classification", in IEEE transactions on instrumentation and measurement, Vol.63 No.2, pp.326-333, 2014.

21. M. Thomas, M. K. Das, and S. Ari, "Automatic ECG arrhythmia classification using dual tree complex wavelet based features" in AEU-International Journal of Electronics and Communications, Vol.69 No.4, pp.715-721, 2015.

22. R. Rodríguez, A. Mexicano, J. Bila, S. Cervantes, and R. Ponce, "Feature Extraction of Electrocardiogram Signals by Applying Adaptive Threshold and Principal Component Analysis" in Journal of Applied Research and Technology, Vol.13 No.2, pp.261-269, 2015.

23. R. G. Afkhami, G. Azarnia, and M. A. Tinati, "Cardiac arrhythmia classification using statistical and mixture modeling features of ECG signals", in Pattern Recognition Letters, Vol. 70, pp. 45-51, 2016

24. H. Li, H. Liang, C. Miao, L. Cao, X. Feng, C. Tang, and E. Li, "Novel ECG signal classification based on KICA nonlinear feature extraction" in Circuits, Systems, and Signal Processing, Vol.35 No.4, pp. 1187-1197, 2016.

25. U. R. Acharya, S. L. Oh, Y. Hagiwara, J. H. Tan, and H. Adeli, "Deep convolutional neural network for the automated detection and diagnosis of seizure using EEG signals", in Computers in biology and medicine, Vol.100, pp.270-278, 2018

26. S. Shadmand, and B. Mashoufi, "A new personalized ECG signal classification algorithm using block-based neural network and particle swarm optimization" in Biomedical Signal Processing and Control, 25, pp.12-23, 2016.

27. R. Varatharajan, G. Manogaran, and M. K. Priyan, "A big data classification approach using LDA with an enhanced SVM method for ECG signals in cloud computing" in Multimedia Tools and Applications, Vol.77 No.8, pp.10195-10215, 2018.

28. S. Padmavathi, and E. Ramanujam, "Naïve Bayes classifier for ecg abnormalities using multivariate maximal time series Motif' Procedia Computer Science, Vol. 47, pp.222-228, 2015.

29. S. Padmavathi, and E. Ramanujam, "Naïve Bayes classifier for ecg abnormalities using multivariate maximal time series Motif' Procedia Computer Science, Vol. 47, pp.222-228, 2015.

30. B. H. Tracey, and E. L. Miller, "Nonlocal means denoising of ECG signals", in IEEE transactions on biomedical engineering, Vol.59 No.9, pp.2383-2386, 2012.

31. S. Abbaspour, H. Gholamhosseini, and M. Linden, "Evaluation of wavelet based methods in removing motion artifact from ECG signal", In 16th Nordic-Baltic Conference on Biomedica Engineering, Cham, Springer, pp.1-4, 2015.

32. O. Singh, and R. K. Sunkaria, "Powerline interference reduction in ECG signals using empirical wavelet transform and adaptive filtering" in Journal of medical engineering \& technology, Vol.39 No.1, pp.60-68, 2015.

33. A. Kumar, G. K. Singh, and S. Anurag, "An optimized cosine-modulated non-uniform filter bank design for sub-band coding of ECG signal" in Journal of King Saud University-Engineering Sciences, Vol.27 No.2, pp.158-169, 2015.

34. M. Sharma, R. San Tan, and U. R. Acharya, "A novel automated diagnostic system for classification of myocardial infarction ECG signals using an optimal biorthogonal filter bank", in Computers in biology and medicine, 102, pp.341-356, 2018.

35. Y. Eminaga, A. Coskun, and I. Kale, "Hybrid IIR/FIR Wavelet Filter Banks for ECG Signal Denoising", in IEEE Biomedical Circuits and Systems Conference (BioCAS), IEEE, pp. 1-4, OCT 2018.

36. O. El B'charri, R. Latif, K. Elmansouri, A. Abenaou, and W. Jenkal, "ECG signal performance de-noising assessment based on threshold tuning of dual-tree wavelet transform" in Biomedical engineering online, Vol.16 No.1, pp.26, 2017.

37. D. Panigrahy, and P. K. Sahu, "Extraction of fetal ECG signal by an improved method using extended Kalman smoother framework from single channel abdominal ECG signal" Australasian physical \& engineering sciences in medicine, Vol.40 No.1, pp.191-207, 2017.

38. Y. Xu, M. Luo, T. Li, and G. Song, "ECG Signal de-noising and baseline wander correction based on CEEMDAN and wavelet threshold" in Sensors, Vol.17 No.12, pp.2754, 2017.

39. H. Hao, M. Liu, P. Xiong, H. Du, H. Zhang, F. Lin, and X. Liu, "Multi-lead model-based ECG signal denoising by guided filter", in Engineering Applications of Artificial Intelligence, Vol.79, pp.34-44, 2019.
40. L. El Bouny, M. Khalil, and A. Adib, "ECG signal filtering based on CEEMDAN with hybrid interval thresholding and higher order statistics to select relevant modes", in Multimedia Tools and Applications, pp.1-23, 2018.

41. M. Bahaz, and R. Benzid, "Efficient algorithm for baseline wanders and powerline noise removal from ECG signals based on discrete Fourier series", in Australasian physical \& engineering sciences in medicine, Vol.41 No.1, pp.143-160, 2018

42. S. Cuomo, R. Farina, and F. Piccialli, "An inverse Bayesian scheme for the denoising of ECG signals", in Journal of Network and Computer Applications, Vol.115, pp.48-58, 2018.

43. H. D. Hesar, and M. Mohebbi, "ECG denoising using marginalized particle extended kalman filter with an automatic particle weighting strategy", in IEEE journal of biomedical and health informatics, Vol.21 No.3, pp.635-644, 2017

44. E. K. Roonizi, and R. Sassi, "A signal decomposition model-based Bayesian framework for ECG components separation" in IEEE Transactions on Signal Processing, Vol.64 No.3, pp.665-674, 2015.

45. P. Nguyen, and J. M. Kim, "Adaptive ECG denoising using genetic algorithm-based thresholding and ensemble empirical mode decomposition" in Information Sciences, Vol. 373, pp.499-511, 2016.

46. G. B. Moody, and R. G. Mark, "The impact of the MIT-BIH arrhythmia database", in IEEE Engineering in Medicine and Biology Magazine, Vol.20 No.3, pp.45-50, 2001.

47. S. Poornachandra, "Wavelet-based denoising using sub-band dependent threshold for ecg signals", in Digital Signal Processing, Vol.18 No.1, pp.49-55, 2008.

48. A. Samit, M. K. Das, and A. Chacko, "ECG signal enhancement using s-transform" in Computers in Biology and Medicine, Vol.43 No.6, pp.649-660, 2013.

49. M. Liu, H. Hao, P. Xiong, F. Lin, Z. Hou, and X. Liu, "Constructing a guided filter by exploiting the butterworth filter for ECG signal enhancement", in Journal of Medical and Biological Engineering, pp.1-13, 2018.

\section{AUTHORS PROFILE}

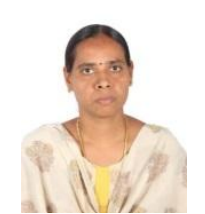

Mythili Thirugnanam is an Associate Professor Senior in the School of Computer Science and Engineering at Vellore Institute of Technology, Vellore, India. She received a Master's in Software Engineering from Vellore Institute of Technology. She has been awarded a doctorate in Computer Science and Engineering at Vellore Institute of Technology in 2014 She has teaching experience of around 12 years. She has research experience of 3 years in handling sponsored projects funded by Govt. of India. Her area of specialization includes Digital Image Processing, Software Engineering, Artificial Intelligence and Machine Learning. She has published more than 40 research papers in international and national journals and presented around 12 papers in various national and international conferences.

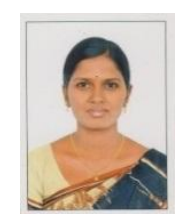

Megana Santhoshi Pasupuleti is a Research Schola in the School of Computer Science and Engineering at Vellore Institute of Technology, Vellore, India. She received her Master's in Computer Science and Engineering from Annamacharya Institute of Technology \& Sciences, JNT University, Anantapur in 2011. Currently working as an Assistant Professor in the Department of Computer Science and Engineering at Chaitanya Bharathi Institute of Technology, JNT University, Anantapur. She has teaching experience of around Four years. Her area of specialization includes Biomedical Signal Processing, Machine Learning, and Internet of Things (IOT). She has published two papers in international journals. 